University of Wollongong

Research Online

Australian Institute for Innovative Materials -

Papers

Australian Institute for Innovative Materials

$1-1-2016$

General synthesis of transition metal oxide ultrafine nanoparticles embedded in hierarchically porous carbon nanofibers as advanced electrodes for lithium storage

\author{
Guanglin Xia \\ University of Wollongong,gx168@uowmail.edu.au \\ Lijun Zhang \\ Fudan University \\ Fang Fang \\ Fudan University \\ Dalin Sun \\ Fudan University \\ Zaiping Guo \\ University of Wollongong, zguo@uow.edu.au
}

See next page for additional authors

Follow this and additional works at: https://ro.uow.edu.au/aiimpapers

Part of the Engineering Commons, and the Physical Sciences and Mathematics Commons

Research Online is the open access institutional repository for the University of Wollongong. For further information contact the UOW Library: research-pubs@uow.edu.au 


\title{
General synthesis of transition metal oxide ultrafine nanoparticles embedded in hierarchically porous carbon nanofibers as advanced electrodes for lithium storage
}

\author{
Abstract \\ A unique general, large-scale, simple, and cost-effective strategy, i.e., foaming-assisted electrospinning, \\ for fabricating various transition metal oxides into ultrafine nanoparticles (TMOs UNPs) that are uniformly \\ embedded in hierarchically porous carbon nanofibers (HPCNFs) has been developed. Taking advantage \\ of the strong repulsive forces of metal azides as the pore generator during carbonization, the formation of \\ uniform TMOs UNPs with homogeneous distribution and HPCNFs is simultaneously implemented. The \\ combination of uniform ultrasmall TMOs UNPs with homogeneous distribution and hierarchically porous \\ carbon nanofibers with interconnected nanostructure can effectively avoid the aggregation, dissolution, \\ and pulverization of TMOs, promote the rapid 3D transport of both $\mathrm{Li}$ ions and electrons throughout the \\ whole electrode, and enhance the electrical conductivity and structural integrity of the electrode. As a \\ result, when evaluated as binder-free anode materials in Li-ion batteries, they displayed extraordinary \\ electrochemical properties with outstanding reversible capacity, excellent capacity retention, high \\ Coulombic efficiency, good rate capability, and superior cycling performance at high rates. More \\ importantly, the present work opens up a wide horizon for the fabrication of a wide range of ultrasmall \\ metal/metal oxides distributed in 1D porous carbon structures, leading to advanced performance and \\ enabling their great potential for promising large-scale applications. \\ Disciplines \\ Engineering | Physical Sciences and Mathematics

\section{Publication Details} \\ Xia, G., Zhang, L., Fang, F., Sun, D., Guo, Z., Liu, H. \& Yu, X. (2016). General synthesis of transition metal \\ oxide ultrafine nanoparticles embedded in hierarchically porous carbon nanofibers as advanced \\ electrodes for lithium storage. Advanced Functional Materials, 26 (34), 6188-6196. \\ Authors \\ Guanglin Xia, Lijun Zhang, Fang Fang, Dalin Sun, Zaiping Guo, Hua-Kun Liu, and Xuebin Yu
}




\section{WILEY-VCH}

DOI: $10.1002 /$ ((please add manuscript number))

Article type: (Full Paper)

\section{General Synthesis of Transition Metal Oxide Ultrafine Nanoparticles Embedded in Hierarchically Porous Carbon Nanofibers as Advanced Electrodes for Lithium Storage}

Guanglin Xia, Lijun Zhang, Fang Fang, Dalin Sun, Zaiping Guo, Huakun Liu, and Xuebin $Y u^{*}$

Dr. G. L. Xia, Dr. L. J. Zhang, Prof. F. Fang, Prof. D. L. Sun, Prof. X. B. Yu

Department of Materials Science, Fudan University, Shanghai, China.

E-mail: yuxuebin@fudan.edu.cn

Dr. G. L. Xia, Prof. Z. P. Guo, Prof. H. K. Liu

Institute for Superconducting and Electronic Materials, University of Wollongong,

Wollongong, Australia.

Keywords: (electrospinning, porous carbon nanofibers, transition metal oxide, nanoparticles, lithium-ion batteries)

A unique general, large-scale, simple, and cost-effective strategy, i.e., foaming-assisted electrospinning, for fabricating various transition metal oxides into ultrafine nanoparticles (TMOs UNPs) that are uniformly embedded in hierarchically porous carbon nanofibers (HPCNFs) has been developed. Taking advantage of the strong repulsive forces of metal azides as the pore generator during carbonization, the formation of uniform TMOs UNPs with homogeneous distribution and HPCNFs was simultaneously implemented. The combination of uniform ultra-small TMOs UNPs with homogeneous distribution and hierarchically porous carbon nanofibers with interconnected nanostructure could effectively avoid the aggregation, dissolution, and pulverization of TMOs, promote the rapid three-dimensional transport of both Li ions and electrons throughout the whole electrode, and enhance the electrical conductivity and structural integrity of the electrode. As a result, when evaluated as binder-free anode materials in Li-ion batteries, they displayed extraordinary electrochemical properties with outstanding reversible capacity, excellent capacity retention, high Coulombic efficiency (CE), good rate capability, and superior cycling performance at high rates. More importantly, the present work opens up a wide horizon for the fabrication of a wide range of ultra-small 


\section{WILEY-VCH}

metal/metal oxides distributed in one-dimensional porous carbon structures, leading to advanced performance and enabling their great potential for promising large-scale applications.

\section{Introduction}

The ever increasing demand for making lithium-ion batteries (LIBs) with larger gravimetric and volumetric capacities, high power density, and long cycle life for various technological applications, e.g., portable electronics, electric vehicles, and renewable energy integration, remains a big challenge. ${ }^{[1-3]}$ The anode material is an indispensable component in determining the capacity, cycle life, and power/energy density of LIBs. Nevertheless, the most intensively used commercial anode material, i.e., graphite, has a low gravimetric capability (theoretically $372 \mathrm{mAh} \mathrm{g}^{-1}$ ), which makes it difficult to meet the demands for high energy density in energy storage systems. Recently, transition metal oxides (TMOs; M: Ti, V, Fe, Zn, Mn, Co, Ni, Mo, etc.) have been intensively exploited as promising anode materials due to their high theoretical specific capacities (> $600 \mathrm{mAh} \mathrm{g}^{-1}$ ), widespread availability, and environmental benignity. ${ }^{[4-24]}$ Unfortunately, the intrinsically low conductivity of TMOs gives them poor rate

performances. ${ }^{[25,26]}$ Furthermore, the biggest issue for TMOs as electrode materials relates to the drastic changes and mechanical strain during the reaction with lithium to form metals/alloys dispersed in the $\mathrm{Li}_{2} \mathrm{O}$ matrix, leading to severe capacity fading after only a few discharge/charge cycles. ${ }^{[27,28]}$

To overcome these limitations, various approaches have been developed to enhance the electrical conductivity while improving the structural stability of TMO-based anode materials. One effective strategy is reducing the size of the particles to the nanoscale, which could shorten the diffusion length for Li ions, leading to high rate capability, and mitigate the absolute strain during lithiation/delithiation, retarding the fracturing and pulverization from significant volume changes. ${ }^{[29-31]}$ Nevertheless, inevitable capacity decay during the 


\section{WILEY-VCH}

electrochemical reaction is usually observed due to the tendency towards aggregation and growth of nanosized particles with high surface energy. Correspondingly, coating or hybridizing nano-TMOs with conductive carbon nanomaterials provides an advanced avenue for enhancing the power and energy densities and initially improving the cycling stability. ${ }^{[32-}$ ${ }^{36]}$ The carbon matrix could act as not only a matrix to enhance the conductivity, but also as a buffer to accommodate the volume changes and prevent particle aggregation during repeated charging-discharging processes, significantly enhancing lithium insertion and extraction. Small and homogeneous particle size, uniform distribution, and porous and robust network structure are definitely the key factors for achieving advanced electrochemical performance. Nonetheless, general and rational design and fabrication of TMO-based anodes with a stabilized structure that can tolerate long cycling are rarely reported and remain a big challenge.

Owing to their uniform structure, orientated electronic and ionic transport, and strong tolerance towards changes in stress, one-dimensional (1D) architectures are capable of displaying fascinating electrochemical properties. ${ }^{[34,35]}$ Recent works have demonstrated that electrospinning is becoming a versatile, simple, cost-effective, and scalable strategy for producing 1D nanostructures, which possess many outstanding properties, including good mechanical strength, excellent flexibility, superior electrical conductivity, and large surface area to volume ratios. ${ }^{[37-41]}$ Nevertheless, a relatively high carbon content resulting from the carbonization of as-electrospun polymers and lack of sufficient porosity could block the diffusion paths of the electrolytes and ions during charge/discharge processes, leading to decreased power and energy. ${ }^{[6,42]}$ Moreover, particles of TMOs tend to grow and aggregate during carbonization of polymeric nanofibers and are usually located on the surfaces of the nanofibers, which is potentially harmful to the formation of a stable solid electrolyte interphase (SEI) layer and results in unsatisfactory electrochemical performance. 


\section{WILEY-VCH}

Herein, we have designed a universal strategy to synthesize transition metal oxides into ultrafine nanoparticles embedded in hierarchically porous carbon nanofibers (TMOs UNPs @ HPCNFs) through a scalable and facile electrospinning strategy with metal azides serving as a pore generator, followed by a simple calcination process. The strong repulsive forces resulting from the intense release of $\mathrm{N}_{2}$ upon the decomposition of metal azides during the simultaneous carbonization process lead to the formation of hierarchically porous carbon nanofibers, which could simultaneously prevent the growth of crystal nuclei during the thermal treatment, thereby resulting in the synthesis of TMOs UNPs (with a typical size of $\sim 4$ $\mathrm{nm}$ ) homogeneously embedded in HPCNFs. By facile changes to the kinds of metal precursors, a series of TMOs UNPs@HPCNFs $(M=\mathrm{Zn}, \mathrm{Mn}$, and Co) have been synthesized, demonstrating the versatile and general nature of this approach. By comparison, without the pore-forming agent, nanoparticles (NPs) of TMOs with a size of over $40 \mathrm{~nm}$ will be formed on the surfaces of the carbon nanofibers (CNFs) as shown in the schematic illustration (Figure 1). The unique 1D nanostructure can effectively suppress the agglomeration and growth of ultra-small TMOs UNPs, improve the electrochemical reaction kinetics, tolerate the tremendous volume changes during repeated charge and discharge processes, and enhance the transport of both electrons and ions due to significantly shortened conducting and diffusion pathways. Moreover, the conductive carbon shells are beneficial for producing a stable SEI layer to protect the electrolyte from persistent decomposition. Furthermore, as-electrospun nanofiber mats grown on copper foil could be directly adopted as electrodes in LIBs without the use of any binder or carbon black, which greatly improves both the energy density and the power density for LIBs, and reduces the cost of the cells. As a consequence, the as-prepared TMOs UNPs@HPCNFs exhibit high specific capacity, excellent rate capability, and cycling stability, which validates their great promise for electrochemical applications in LIBs.

\section{Results and Discussion}


Figure 1 illustrates the fabrication process for TMOs UNPs@HPCNFs ( $M=\mathrm{Zn}, \mathrm{Mn}$, and Co) by the porogen-assisted electrospinning strategy. Specifically, the polymeric nanofibers containing the various metal precursors and the pore generator $\left(\mathrm{LiN}_{3}\right)$ were first electrospun onto copper foil and then dewatered under dynamic vacuum at $75{ }^{\circ} \mathrm{C}$. Subsequently, the dried nanofibers were further annealed in flowing $\mathrm{N}_{2}$ at $550{ }^{\circ} \mathrm{C}$ for $3 \mathrm{~h}$ with a heating rate of $2{ }^{\circ} \mathrm{C}$ $\min ^{-1}$. During the carbonization process, $\mathrm{LiN}_{3}$ will simultaneously undergo a violent decomposition process to form a strong vapor phase, which leads to the formation of abundant mesopores and macropores inside the polymeric nanofibers ${ }^{[43]}$ and functions as a separator to prevent the TMO nanocrystals from agglomerating and growing into larger particles.

The morphology and nano-architecture of the as-spun polymeric nanofibers and obtained TMOs UNPs@HPCNFs after carbonization were characterized by SEM and TEM coupled with energy-dispersive X-ray spectroscopy (EDS). Typical digital photographs of the as-spun polymer films (left, white) and the obtained TMOs UNPs@HPCNFs films (right, black) after annealing are displayed in Figure 2a. The film with diameter of $1.5 \mathrm{~cm}$ is integral and has no cracks (Figure S1, ESI†), showing the structural integrity of the network and its potential application in LIBs without the need for introducing any supplementary components. An SEM image of the cross-linked TMOs UNPs@HPCNFs hybrid film from a side view shows that the thickness of the nanostructured layers is approximately $200 \mu \mathrm{m}$ (Figure 2b), and the nanofibers are randomly oriented, forming the architecture of a three-dimensional (3D) porous cross-linked network. Good contact between the HPCNFs and the collector, and the 3D continuous distribution result in efficient charge transfer between the current collector and the electroactive materials. Interestingly, the as-electrospun nanofibers (Figure $2 \mathrm{c}$ and $\mathrm{d}$ ) present the typical appearance of electrospun fibers with smooth surfaces and diameters of around $200 \mathrm{~nm}$, while abundant mesopores and macropores are observed on the surfaces of the obtained TMOs UNPs@HPCNFs (Figure 2e). By comparison, although the samples 


\section{WILEY-VCH}

electrospun without the presence of $\mathrm{LiN}_{3}$ preserve a similar 1D fibrous morphology (Figure $\mathrm{S} 2$ ), no porous structures are observed on the surfaces of the as-prepared CNFs (Figure 2f).

The morphology of the as-prepared TMOs UNPs@HPCNFs was further characterized by TEM techniques (Figure 3). It could be clearly observed that abundant interconnected pores, including mesopores and macropores, are uniformly distributed in the long, continuous, and interconnected CNF matrix. With the increase of contents of $\mathrm{LiN}_{3}$ in the precursors, the intensity and pores increased (Figure S3) under the same synthetic conditions. After adding excess $\mathrm{LiN}_{3}$, the as-prepared TMOs UNPs@HPCNFs, however, tends to aggregate with inhomogenous structure. It demonstrates that $\mathrm{LiN}_{3}$ plays an important role in the generation of hierarchical pores and appropriate concentration of $\mathrm{LiN}_{3}$ is essential for obtaining the unifom carbon nanofibers. The porous texture of TMOs UNPs@HPCNFs was further investigated by nitrogen adsorption/desorption isotherms at $77 \mathrm{~K}$ (Figures S4-S6), which exhibit typical typeIV behavior with a distinct hysteresis loop at relative pressures $P / P_{0}$ ranging from 0.5 to 1 , suggesting the presence of a large number of mesopores. Based on the Brunauer-EmmettTeller (BET) method, a specific surface area of 116,89 , and $63 \mathrm{~m}^{2} \mathrm{~g}^{-1}$ and a large pore volume of $0.53,0.39$, and $0.37 \mathrm{~cm}^{3} \mathrm{~g}^{-1}$ is revealed for $\mathrm{ZnO}$ UNPs@HPCNFs, MnO UNPs@HPCNFs, and CoO UNPs@HPCNFs, respectively. The relative pore size distributions (Figures S4-S6) determined from the adsorption branches exhibit many peaks in the range from 10 to $100 \mathrm{~nm}$, which confirms the porous characteristics of the HPCNFs and agrees well with the SEM/TEM observations. The macropores and mesopores on the surface and throughout the nanofibers promote the rapid access of lithium ions to interact with the active materials, contributing to good rate capability and buffering the volumetric expansion during repeated charging and discharging cycles. More importantly, the magnified TEM images (Figure 3d, e, and f) reveal that individual $\mathrm{ZnO}, \mathrm{MnO}$, and $\mathrm{CoO}$ UNPs (black dots) with an average particle size of only $4 \mathrm{~nm}$ were homogeneously dispersed in the HPCNFs. In addition, no aggregated TMO UNPs were found on the surface. Therefore, the as-formed 


\section{WILEY-VCH}

HPCNFs could not only effectively prevent the aggregation of TMO UNPs, but also avoid the exfoliation of the TMO UNPs during the repeated charging and discharging cycles, preserving the integrity of the whole electrode. By comparison, the mean particle size of the TMOs is over $40 \mathrm{~nm}$, and the particles are anchored on the surfaces of the CNFs (Figure 2f) after carbonization of the as-electrospun nanofibers without $\mathrm{LiN}_{3}$ (denoted as TMOs NPs@CNFs $(\mathrm{M}=\mathrm{Zn}, \mathrm{Mn}$, and $\mathrm{Co}))$. This suggests that well distributed $\mathrm{LiN}_{3}$ could act as an efficient pore generator, leading to uniform formation of abundant mesopores and macropores created by the repulsive release of $\mathrm{N}_{2}$, leading to increased surface area of the porous carbon matrices, which could both effectively facilitate the dispersion of TMO nanograins and prevent the growth and agglomeration of TMO UNPs during thermal treatment.

The representative selected-area electron diffraction (SAED) patterns (insets of Figure 3g, h, and i) demonstrate the poor crystallinity of the TMO UNPs inside the HPCNFs, which corresponds well with the X-ray diffraction (XRD) results (Figure 4a). Due to the ultra-small size of the obtained $\mathrm{ZnO}, \mathrm{MnO}$ and $\mathrm{CoO}$ UNPs, the corresponding XRD peaks exhibited weakly broadened lines. High-resolution TEM (HRTEM) images (Figure 3g, h and i) confirm that the weak measured lattice spacing in ZnO UNPs@HPCNFs, MnO UNPs@HPCNFs, and CoO UNPs@ HPCNFs is calculated to be $0.26,0.26$, and $0.21 \mathrm{~nm}$, respectively, corresponding to the (002) plane spacing of $\mathrm{ZnO}$, (111) plane spacing of $\mathrm{MnO}$, and (200) plane spacing of $\mathrm{CoO}$, respectively. In addition, no obvious lattice fringes of carbon are detected, indicating the amorphous nature of the carbon, which is in good agreement with the XRD results, with no intensive peak indexed to carbon. ${ }^{[44]}$ The Raman spectra of the TMOs UNPs@HPCNFs (Figure 4b) further indicates the presence of two broad peaks at 1321 and $1582 \mathrm{~cm}^{-1}$, corresponding to the typical disorder (D) band and graphite (G) band of amorphous carbon, respectively. Due to the abundant defects and vacancies of amorphous carbon, the diffusion of lithium ions could be favored, and more reversible active sites for Li storage would be available in the as-prepared HPCNFs, which would contribute to the overall capacity. ${ }^{[45,46]}$ 


\section{WILEY-VCH}

Moreover, scanning TEM (STEM) with the corresponding elemental mapping images (Figure $3 \mathrm{j}, \mathrm{k}$, and $\mathrm{l}$ ) validates that the $\mathrm{Zn}, \mathrm{Mn}$, and Co elements are homogeneously distributed inside the HPCNFs. The content of carbon in the TMOs UNPs@HPCNFs is determined to be around 30.9, 29.9, and 33.2 wt.\% for $\mathrm{ZnO}, \mathrm{MnO}$, and $\mathrm{CoO}$ UNPs@HPCNFs, respectively, based on the TGA results (Figure 4c). The weight increase of MnO UNPs@HPCNFs from $500{ }^{\circ} \mathrm{C}$ to $600{ }^{\circ} \mathrm{C}$ could be attributed to the oxidation of $\mathrm{MnO}$ to $\mathrm{Mn}_{2} \mathrm{O}_{3} .{ }^{[9]}$

XPS was further employed to analyze the surface electronic states and the compositions of the products. It revealed the presence of carbon, nitrogen, and oxygen, and also exhibited the relevant characteristic metal peaks in the as-synthesized TMOs UNPs@HPCNFs (Figure 4d, e, and f). The high-resolution C 1s spectrum of ZnO UNPs@HPCNFs (Figure S7a) demonstrates the formation of $\mathrm{C}-\mathrm{N}$ and $\mathrm{C}=\mathrm{N}$ bonds, and the formation of $\mathrm{Li}_{2} \mathrm{CO}_{3}$ without the apperance of $\mathrm{Li}_{3} \mathrm{~N}$ was demonstrated after the calcination of the as-electrospun nanofibers without washing (Figure S8). Therefore, the presence of N in TMOs UNPs@HPCNFs could be attributed to the reaction between $\mathrm{Li}_{3} \mathrm{~N}$ and as-electrospun nanofibers during the calcination process. Furthermore, the high-resolution N 1s spectrum of ZnO UNPs@HPCNFs (Figure S7b) exhibits four fitted peaks centered at $398.4 \mathrm{eV}, 399.8 \mathrm{eV}, 401.1 \mathrm{eV}$, and 402.1 $\mathrm{eV}$, which are attributed to pyridinic $\mathrm{N}$, pyrrolic $\mathrm{N}$, graphitic $\mathrm{N}$, and oxidized $\mathrm{N}$, respectively. It further verifies the doping of $\mathrm{N}$ into HPCNFs, which could facilitate the electron transfer and Li ions diffusion kinetics. ${ }^{[47]}$ The characteristic peaks of the metals were further examined by high-resolution XPS analysis (insets of Figure 4d, e, and f), in which two signals attributed to the $2 \mathrm{p}_{3 / 2}$ and $2 \mathrm{p}_{1 / 2}$ orbitals for $\mathrm{Zn}(\mathrm{II}), \mathrm{Mn}(\mathrm{II})$, and $\mathrm{Co}(\mathrm{II})$, respectively, could be clearly observed, confirming the formation of the corresponding TMOs in the HPCNFs. These results obviously demonstrate that our strategy to synthesize TMOs UNPs@HPCNFs is universal and efficient, and it has several key advantages for achieving advanced electrochemical performance, including ultra-small TMO UNPs with homogeneous particle size and uniform 


\section{WILEY-VCH}

distribution, and a porous and robust network structure, which are integrated into the asprepared TMOs UNPs@HPCNFs.

Such an ultra-uniform distribution of TMOs UNPs in the HPCNFs, in association with the unique 1D nanostructures with hierarchically porous structure, was expected to lead to high capacity and excellent performance when the samples were evaluated for lithium storage. To confirm this, the ZnO UNPs@HPCNFs sample was selected and investigated in detail as a typical sample of these electroactive materials in LIBs. It should be noted that all the specific capacity is calculated based on the total mass of the TMOs UNPs@HPCNFs, and the loading amount of the active material for the electrode is around $1.6 \mathrm{mg} \mathrm{cm}^{-2}$, which can be controlled by altering the electrospinning time. Figure 5a shows typical cyclic voltammograms (CVs) of ZnO UNPs@HPCNFs in the initial three cycles at a scan rate of $0.1 \mathrm{mV} \mathrm{s}^{-1}$ between 0.001 and $3 \mathrm{~V}$. During the first cathodic sweep, the wide peak at $0.5-0.75 \mathrm{~V}$ could be ascribed to the reduction of $\mathrm{ZnO}$ to $\mathrm{Zn}$ and the alloying reaction between $\mathrm{Li}$ and $\mathrm{Zn}$, as well as the formation and growth of the SEI layer. ${ }^{[48]}$ The anodic curves display two peaks, in which the peak at $0.51 \mathrm{~V}$ is related to the multi-step de-alloying process of $\mathrm{Li}-\mathrm{Zn}$ alloy, and the broad peak at 1.37 $\mathrm{V}$ is related to the formation of $\mathrm{ZnO}$ according to the redox reaction between $\mathrm{Zn}$ and $\mathrm{Li}_{2} \mathrm{O}{ }^{[49,50]}$ Therefore, the overall reaction process of ZnO UNPs@ $@$ HCNFs in LIBs could be described as Equations 1, 2:

$$
\begin{aligned}
& \mathrm{ZnO}+2 \mathrm{Li}^{+}+2 \mathrm{e}^{-1} \leftrightarrow \mathrm{Zn}+\mathrm{Li}_{2} \mathrm{O} \\
& \mathrm{Zn}+\mathrm{Li}^{+}+\mathrm{e}^{-1} \leftrightarrow \mathrm{LiZn}
\end{aligned}
$$

In order to verify the reaction mechanism of ZnO UNPs@HPCNFs, HRETM of ZnO UNPs@HPCNFs at different discharged states was subsequently conducted. It can be clearly seen that the discharged product at discharging to $0.55 \mathrm{~V}$ (Figure S9a) is well attributed to $\mathrm{Zn}$ with the (101) interplanar spacing of $0.21 \mathrm{~nm}$. When the electrode is further discharged to $0.01 \mathrm{~V}$ (Figure S9b), lattice fringes with the distance of $0.22 \mathrm{~nm}$, which is in agreement with (220) plane of LiZn, were observed. The above observations demonstrate the reversible 


\section{WILEY-VCH}

reaction mechanism through electrochemical conversion of eqs 1 and 2. From the second cycle onwards, the CV curves are almost superimposed, indicating excellent reversibility of the electrochemical reactions.

Figure 5b presents the voltage profiles of ZnO UNPs@HPCNFs for different cycles between 0.001 and $3 \mathrm{~V}$ at a current density of $0.1 \mathrm{~A} \mathrm{~g} \mathrm{~g}^{-1}$, which exhibit typical characteristics of a $\mathrm{ZnO}$ electrode. ${ }^{[5]}$ Impressively, the first discharge and charge capacities are 1923 and $1369 \mathrm{mAh} \mathrm{g}^{-1}$, respectively, corresponding to a coulombic efficiency (CE) of $71 \%$. This CE is generally regarded as high, which indicates that the encapsulation of ZnO UNPs in HPCNFs could considerably alleviate detrimental reactions between the $\mathrm{ZnO}$ and the electrolyte. ${ }^{[51]}$ The capacity loss during the first cycle is mainly attributed to the incomplete conversion reaction and irreversible lithium loss due to the formation of a solid-electrolyte interphase (SEI) layer on the surface of the ZnO UNPs@HPCNFs. It should be noted that HPCNFs, which is prepared in the similar method of synthesizing the ZnO UNPs@HPCNFs without adding $\mathrm{Zn}\left(\mathrm{CH}_{3} \mathrm{COO}\right)_{2} \cdot 2 \mathrm{H}_{2} \mathrm{O}$ in the precursor, contribute about $560 \mathrm{mAh} \mathrm{g}^{-1}$ at $0.1 \mathrm{~A} \mathrm{~g}^{-1}$ (Figure S10), considering the theoretical Li-storage capacity of $\mathrm{ZnO}\left(987 \mathrm{mAh} \mathrm{g}^{-1}\right.$ ), the theoretical capacity of ZnO UNPs@HPCNFs is calculated to be $855 \mathrm{mAh} \mathrm{g}^{-1}$ as follows:

$$
\begin{gathered}
\mathrm{C}_{\mathrm{ZnO} \text { UNPs } @ \mathrm{HPCNF}}=\mathrm{C}_{\mathrm{ZnO}} \times \%_{\text {mass of ZnO }}+\mathrm{C}_{\mathrm{HPCNFs}} \times \%_{\text {mass of HPCNFs }} \\
=987 \times 69.1 \%+560 \times 30.9 \%=855 \mathrm{mAh} \mathrm{g}^{-1}
\end{gathered}
$$

Figure 5c shows the cycling performance for the ZnO UNPs@HPCNFs at the constant current density of $0.1 \mathrm{~A} \mathrm{~g}^{-1}$. The anode displays excellent capacity retention, delivering a discharge capacity of $1136 \mathrm{mAh} \mathrm{g}^{-1}$ after 150 cycles without any apparent capacity loss, which is actually $32.9 \%$ higher than the theoretical capacity of ZnO UNPs@HPCNFs, indicating the significant synergistic coupling effect between ultrasmall $\mathrm{ZnO}$ nanoparticles and HPCNFs. Moreover, it should be pointed out that a high CE of over 99\% was achieved after only a few cycles, demonstrating the high reversibility and stability of the $\mathrm{ZnO}$ UNPs@HPCNFs. 


\section{WILEY-VCH}

The ZnO UNPs@HPCNFs electrode was further cycled at various current densities to investigate the rate performance. The corresponding discharge capacities at various current densities from 0.1 to $3 \mathrm{~A} \mathrm{~g}^{-1}$ demonstrate the much improved rate capability for the $\mathrm{ZnO}$ UNPs@HPCNFs (Figure 5d). At a low current density of $0.1 \mathrm{~A} \mathrm{~g}^{-1}$, the specific reversible capacity could reach $\sim 1214 \mathrm{mAh} \mathrm{g}^{-1}$. Notably, even at a higher current density of $3 \mathrm{~A} \mathrm{~g}^{-1}$, the electrode still delivers a capacity of $\sim 618 \mathrm{mAh} \mathrm{g}^{-1}$, which is much higher than the practical capacity of commercial graphite and those of $\mathrm{ZnO}$ NPs@CNFs and $\mathrm{ZnO}$ powders. More importantly, when the current density decreases to $0.1 \mathrm{~A} \mathrm{~g}^{-1}$ after cycling under high current densities, the ZnO UNPs@HPCNFs exhibits very stable cyclability, and it could deliver a reversible capacity of $1190 \mathrm{mAh} \mathrm{g}^{-1}$ with capacity fading of only $2 \%$ compared with the relative capacity obtained in the initial cycle at $0.1 \mathrm{~A} \mathrm{~g}^{-1}$. Additionally, it could be observed, after a slight decrease of the reversible capacity to $525 \mathrm{mAh} \mathrm{g}^{-1}$ in the initial 300 cycles, a specific capacity as high as $630 \mathrm{mAh} \mathrm{g}^{-1}$ could be obtained at the current density of $3 \mathrm{~A} \mathrm{~g}^{-1}$ even after 500 deep charging and discharging cycles (Figure 5e), which further attests to the excellent cycling stability of ZnO UNPs@HPCNFs. The gradual increasing of capacity during cycling process was generally attributed to the gradual decomposition of the irreversible $\mathrm{Li}_{2} \mathrm{O}$ formed in the $1^{\text {st }}$ discharge process, the interfacial lithium storage and/or the wetting problem between the electrode and electrolytes, which will result in a gradually increased utilization of active materials upon continuous cycling. ${ }^{[12,52]}$ The minor capacity fluctuation during the long-cycling test could be attributed to the unstability of the SEI film, the electrolyte degradation, the reaction of oxygen-containing functional groups on the carbon with lithium ions, and/or the small temperature fluctuation of the environment. ${ }^{[53]}$

To further understand the outstanding electrochemical performance of $\mathrm{ZnO}$ UNPs@HPCNFs, TEM analysis (Figure 5f) was conducted to examine the morphological changes in the electrode after 500 charge/discharge cycles at $3 \mathrm{~A} \mathrm{~g}^{-1}$. The TEM image obviously confirms that the porous structure of the carbon nanofibers is still well preserved 


\section{WILEY-VCH}

after cycling for the ZnO UNPs@HPCNFs, which demonstrates that the tailored nanostructure of the TMOs UNPs@HPCNFs could effectively restrain the pulverization and aggregation of TMO nanograins during continuous lithiation and delithiation processes, thus ensuring high cycling stability.

Electrochemical impedance spectroscopy (EIS) measurements on the electrodes containing ZnO UNPs@HPCNFs, ZnO NPs@CNFs, and commercial ZnO powders were also performed, both before cycling and after 500 cycles, to gain deeper insight into the remarkably enhanced electrochemical reaction kinetics and cycling performance of the ZnO UNPs@HPCNFs. As shown in Figure S11, all the Nyquist plots present a depressed semicircle in the medium frequency region, which is associated with internal resistances in the electrode. Clearly, the charge transfer resistance of ZnO UNPs@HPCNFs is the lowest, and it is much lower than those of ZnO NPs@CNFs and ZnO powders, representing the highly enhanced electrical conductivity and electrochemical reaction kinetics of the whole electrode. More importantly, the diameter of the semicircle for the ZnO UNPs@HPCNFs at high frequency does not increase much, even after 500 cycles, thus suggesting the formation of a stable SEI layer on the surface of the electrode in the initial cycles and high structural integrity. ${ }^{[19]}$

The cycling performance of MnO UNPs@HPCNFs and CoO UNPs@HPCNFs at a constant current density of $0.1 \mathrm{~A} \mathrm{~g}^{-1}$ was further evaluated (Figure $5 \mathrm{~g}$ ), the results of which clearly authenticate their superior cyclability, delivering the discharge capacity of 1118 and $1057 \mathrm{mAh} \mathrm{g}^{-1}$, respectively, at the end of 100 charge-discharge cycles. By comparison, only a reversible capacity of 104 and $117 \mathrm{mAh} \mathrm{g}^{-1}$ after 100 cycles was observed for pure $\mathrm{MnO}$ and $\mathrm{ZnO}$, respectively, coupled with rapid capacity fading. Remarkably, the CE approaches $100 \%$ for both MnO UNPs@HPCNFs and CoO UNPs@HPCNFs, suggesting that the superior cycling stability for reversible $\mathrm{Li}^{+}$storage is highly repeatable. These results obviously validate the general practicability of this unique nanostructure for modifying the electrochemical performance of TMOs as anodes. 


\section{WILEY-VCH}

The exceptional electrochemical performance of TMOs UNPs@HPCNFs with ultra-high specific capacity, excellent rate capability, and ultra-long cycle life could be attributed to the following merits. First, the formation of ultra-small TMOs UNPs $(\sim 4 \mathrm{~nm})$ could significantly shorten the solid-state diffusion of $\mathrm{Li}^{+}$and mitigate the volume changes and agglomeration caused by lithium insertion/extraction. Moreover, the interconnected 1D morphology of the HPCNFs directly grown on the copper foil can serve to provide channels for fast electron transport, which could significantly increase the electrical conductivity of the uniformly distributed TMO UNPs and decrease the internal resistance of the LIBs, thereby resulting in a high specific capacity. Furthermore, the plentiful mesopores and macropores inside the HPCNFs provide sufficient void space for the volume changes during charging and discharging processes, which is beneficial for maintaining the structural integrity and storing the electrolyte in "reservoirs", which significantly shorten the diffusion pathways of Li ions and increase the contact interface between the active materials and the electrolyte, thus facilitating rapid mass and electron transport and high-rate capability. The hierarchical porous carbon nanofibers could facilitate the three-dimensional fast diffusion of electrolyte, restrict the growth of the SEI layer, and provide a sturdy shell to mechanically accommodate the stress associated with the large volume changes during electrochemical reactions of the ultrasmall TMO UNPs, all of which promote cycling stability. All of these features are responsible for the superior high-rate cycling performance of the TMOs UNPs@HPCNFs.

\section{Conclusion}

In summary, we have developed a novel, scalable, and general route to synthesize ultra-small TMO UNPs homogenously distributed in hierarchically porous carbon nanofibers by adopting the electrospinning technique. Metal azide was first utilized as the pore-forming agent to create abundant mesopores and macropores inside the HPCNFs, which led to the simultaneous formation of TMO UNPs with homogeneous distribution. This unique structure represents a significant improvement in the electrochemical performance of TMOs as anode 


\section{WILEY-VCH}

materials for LIBs, e.g., an ultra-high reversible capacity of $1136 \mathrm{mAh} \mathrm{g}^{-1}$ was obtained for ZnO UNPs@HPCNFs after 150 cycles at $0.1 \mathrm{~A} \mathrm{~g}^{-1}$ and, more importantly, the reversible capacity at a high current rate of $3 \mathrm{~A} \mathrm{~g}^{-1}$ still approached $610 \mathrm{mAh} \mathrm{g}^{-1}$ after 500 cycles. Furthermore, the present method is facile, large-scale, and generally applicable for the synthesis of TMOs UNPs@HPCNFs, and it offers a new, scalable, and general pathway for the rational design of functional materials for a broad range of applications such as catalysis, nanoelectronics, and energy storage or conversion.

\section{Experimental Section}

Materials Synthesis: Poly(vinyl alcohol) (PVA, 0.5 g, Alfa Aesar, Mv = 88000) was mixed with deionized water $(5 \mathrm{~mL})$ and stirred in a water bath at $90{ }^{\circ} \mathrm{C}$ for $8 \mathrm{~h}$ to make a homogeneous PVA solution. After cooling down to room temperature, $\mathrm{LiN}_{3}$ solution $(0.4 \mathrm{~mL}$, 20 wt.\% in water) and $\mathrm{Mn}\left(\mathrm{CH}_{3} \mathrm{COO}\right)_{2} \cdot 4 \mathrm{H}_{2} \mathrm{O}, \mathrm{Zn}\left(\mathrm{CH}_{3} \mathrm{COO}\right)_{2} \cdot 2 \mathrm{H}_{2} \mathrm{O}$, or $\mathrm{Co}\left(\mathrm{CH}_{3} \mathrm{COO}\right)_{2} \cdot 2 \mathrm{H}_{2} \mathrm{O}$ (100 mg, Sigma) were then added and vigorously stirred for $6 \mathrm{~h}$ to make a homogeneous spinning dope. The resultant precursor solution was poured into a syringe with an 18-gauge blunt-tip needle. The flow rate of solution was approximately $300 \mu \mathrm{L} \mathrm{h}^{-1}$, controlled by a syringe pump (Longer, TJP-3A, China), and a grounded stainless steel plate was horizontally placed $10 \mathrm{~cm}$ from the needle to collect the as-electrospun nanofibers. A high voltage of 15 $\mathrm{kV}$ was applied by a high-voltage power supply. The as-collected fibers were firstly dewatered at a temperature of $75{ }^{\circ} \mathrm{C}$ under dynamic vacuum for $15 \mathrm{~h}$ and then calcined at $550{ }^{\circ} \mathrm{C}$ for $3 \mathrm{~h}$ with a heating rate of $2{ }^{\circ} \mathrm{C} \min ^{-1}$ under dynamic $\mathrm{N}_{2}$ atmosphere to obtain the TMOs UNPs@HPCNFs after washing and vacuum-drying at $80{ }^{\circ} \mathrm{C}$.

Materials Characterization: Nitrogen absorption/desorption isotherms (Brunauer-EmmettTeller (BET) technique) at the temperature of liquid nitrogen were collected via a Quantachrome NOVA 4200e instrument to characterize the pore structure of the samples. The crystalline phase of the samples was also detected by X-ray photoelectron spectroscopy (XPS, Surface Science Instruments S-probe spectrometer). Raman spectra were collected at ambient 


\section{WILEY-VCH}

temperature with a NEXUS 670 FT-IR Raman spectrometer. The phase composition of the powdery composite was analysed by XRD (D8 Advance, Bruker AXS) with $\mathrm{Cu} \mathrm{K} \alpha$ radiation. Thermal property measurements were performed by thermogravimetric analysis (TGA, STA 409C) with a heating rate of $10{ }^{\circ} \mathrm{C} \mathrm{min}^{-1}$ under air. The microstructures were observed by transmission electron microscopy (TEM, JEM-2100F, JEOL) and field-emission scanning electron microscopy (SEM, S-4800, Hitachi).

Electrochemical Measurements: Electrochemical measurements were performed using coin type 2032 cells. The working electrode was prepared as follows. The TMOs UNPs@HPCNFs networks were directly used as binder-free anodes. For the conventional samples, TMOs, carbon black, and poly(vinyl difluoride) (PVDF) were mixed in N-methyl-2-pyrrolidone (NMP) with a weight ratio of 80:10:10, and then the slurry was pasted onto pure $\mathrm{Cu}$ foil. The electrolyte consisted of a solution of $1 \mathrm{M} \mathrm{LiPF}_{6}$ in ethylene carbonate (EC)/dimethyl carbonate (DMC) (volume ratio of 1:1). Pure lithium foil was used as the counter electrode, and the separator was a polypropylene membrane from Celgard. The coating thickness on $\mathrm{Cu}$ foil was about $100 \mu \mathrm{m}$. The cells were assembled in an argon-filled glove box. The charge and discharge performances were investigated on a LAND-CT2001C test system within the range of $0.001-3 \mathrm{~V}$ at different current densities. Cyclic voltammetry (CV) was performed on an Autolab PGSTAT302N electrochemical workstation at a scan rate of $0.1 \mathrm{mV} \mathrm{s}^{-1}$.

\section{Supporting Information}

Supporting Information is available from the Wiley Online Library or from the author.

\section{Acknowledgements}

This work was partially supported by the National Natural Science Foundation of China (21271046, 51471053, and 51571063). We thank Dr. Tania Silver for critical reading of the manuscript. 


\section{WILEY-VCH}

[1] G. Zhang, H. B. Wu, H. E. Hoster, X. W. Lou, Energy Environ. Sci. 2014, 7, 302.

[2] C. Yuan, H. B. Wu, Y. Xie, X. W. Lou, Angew. Chem. Int. Ed. 2014, 53, 1488.

[3] B. Dunn, H. Kamath, J. M. Tarascon, Science 2011, 334, 928.

[4] L. Zhang, H. B. Wu, X. W. Lou, Adv. Energy Mater. 2014, 4, 1300958.

[5] G. Zhang, S. Hou, H. Zhang, W. Zeng, F. Yan, C. C. Li, H. Duan, Adv. Mater. 2015, $27,2400$.

[6] F. Zhang, C. Yuan, J. Zhu, J. Wang, X. Zhang, X. W. Lou, Adv. Funct. Mater. 2013, $23,3909$.

[7] G. Zhou, D. W. Wang, L. C. Yin, N. Li, F. Li, H. M. Cheng, ACS Nano 2012, 6, 3214.

[8] Y. Shi, B. Guo, S. A. Corr, Q. Shi, Y. S. Hu, K. R. Heier, L. Chen, R. Seshadri, G. D. Stucky, Nano Lett. 2009, 9, 4215.

[9] H. Jiang, Y. Hu, S. Guo, C. Yan, P. S. Lee, C. Li, ACS Nano 2014, 8, 6038.

[10] B. Liu, X. Hu, H. Xu, W. Luo, Y. Sun, Y. Huang, Sci. Rep. 2014, 4, 4229.

[11] W. Li, F. Wang, Y. Liu, J. Wang, J. Yang, L. Zhang, A. A. Elzatahry, D. Al-Dahyan, Y. Xia, D. Zhao, Nano Lett. 2015, 15, 2186.

[12] G. Xu, Y. Li, T. Ma, Y. Ren, H. Wang, L. Wang, J. Wen, D. Miller, K. Amine, Z. H. Chen. Nano Energy 2015, 18, 253.

[13] H. Kim, M. G. Kim, J. Cho, Adv. Energy Mater. 2012, 2, 1425.

[14] X. Xu, R. Cao, S. Jeong, J. Cho, Nano Lett. 2012, 12, 4988.

[15] C. Wu, J. Maier, Y. Yu, Adv. Funct. Mater. 2015, 25, 3488.

[16] Q. An, F. Lv, Q. Liu, C. Han, K. Zhao, J. Sheng, Q. Wei, M. Yan, L. Q. Mai, Nano Lett. 2014, 14, 6250.

[17] H. P. Cong, S. Xin, S. H. Yu, Nano Energy 2015, 13, 482.

[18] Z. S. Wu, W. Ren, L. Wen, L. Gao, J. Zhao, Z. Chen, G. Zhou, F. Li, H. M. Cheng, ACS Nano 2010, 4, 3187. 


\section{WILEY-VCH}

[19] L. Xia, S. Q. Wang, G. X. Liu, L. X. Ding, D. D. Li, H. H. Wang, S. Z. Qiao, Small 2016, 12,853 .

[20] Y. Zou, Y. Wang, Nanoscale 2011, 3, 2615.

[21] W. Li, F. Wang, S. Feng, J. Wang, Z. Sun, B. Li, Y. Li, J. Yang, A. A. Elzatahry, Y. Y. Xia, D. Y. Zhao, J. Am. Chem. Soc. 2013, 135, 18300.

[22] L. Zhang, H. B. Wu, B. Liu, X. W. Lou, Energy Environ. Sci. 2014, 7, 1013.

[23] X. Y. Yu, H. B. Wu, F. X. Ma, X. W. Lou, Angew. Chem. Int. Ed. 2015, 54, 4001.

[24] B. Y. Guan, L. Yu, J. Li, X. W. Lou, Sci. Adv. 2016, 2, e1501554.

[25] J. Jiang, Y. Li, J. Liu, X. Huang, C. Yuan, X. W. Lou, Adv. Mater. 2012, 24, 5166.

[26] R. S. Devan, R. A. Patil, J. H. Lin, Y. R. Ma, Adv. Funct. Mater. 2012, 22, 3326.

[27] L. Ji, Z. Lin, M. Alcoutlabi, X. Zhang, Energy Environ. Sci. 2011, 4, 2682.

[28] W. Wei, Z. Wang, Z. Liu, Y. Liu, L. He, D. Chen, A. Umar, L. Guo, J. Li, J. Power Sources 2013, 238, 376.

[29] K. T. Lee, J. Cho, Nano Today 2011, 6, 28.

[30] J. N. Tiwari, R. N. Tiwari, K. S. Kim, Prog. Mater. Sci. 2012, 57, 724.

[31] M. J. Armstrong, C. O’Dwyer, W. J. Macklin, J. D. Holmes, Nano Res. 2013, 7, 1.

[32] Y. Tang, Y. Zhang, W. Li, B. Ma, X. Chen, Chem. Soc. Rev. 2015, 44, 5926.

[33] B. Luo, L. Zhi, Energy Environ. Sci. 2015, 8, 456.

[34] L. Zhang, G. Zhang, H. B. Wu, L. Yu, X. W. Lou, Adv. Mater. 2013, 25, 2589.

[35] H. B. Wu, G. Q. Zhang, L. Yu, X. W. Lou, Nanoscale Horiz. 2016, 1, 27.

[36] C. He, S. Wu, N. Zhao, C. Shi, E. Liu, J. Li, ACS Nano 2013, 7, 4459.

[37] S. Ding, J. S. Chen, X. W. Lou, Adv. Funct. Mater. 2011, 21, 4120.

[38] Y. Liu, N. Zhang, L. F. Jiao, J. Chen, Adv. Mater. 2015, 27, 6702.

[39] S. J. Peng, G. Jin, L. L. Li, K. Li, M. Srinivasan, S. Ramakrishna, J. Chen, Chem. Soc. Rev. 2016, 45, 1225.

[40] C. L. Zhang, S. H. Yu, Chem. Soc. Rev. 2014, 43, 4423. 


\section{WILEY-VCH}

[41] J. Wu, N. Wang, Y. Zhao, L. Jiang, J. Mater. Chem. A 2013, 1, 7290.

[42] L. Zhao, Y. S. Hu, H. Li, Z. Wang, L. Chen, Adv. Mater. 2011, 23, 1385.

[43] G. L. Xia, D. Li, X. Chen, Y. Tan, Z. Tang, Z. Guo, H. Liu, Z. Liu, X. B. Yu, Adv. Mater. 2013, 25, 6238.

[44] J. Hwang, S. H. Woo, J. Shim, C. Jo, K. T. Lee, J. Lee, ACS Nano 2013, 7, 1036.

[45] Y. C. Liu, N. Zhang, L. F. Jiao, J. Chen, Adv. Mater. 2015, 27, 6702.

[46] Z. Zhu, F. Cheng, J. Chen, J. Mater. Chem. A 2013, 1, 9484.

[47] L. Qie, W. Chen, Z. Wang, Q. Shao, X. Li, L. Yuan, X. Hu, W. Zhang, Y. H. Huang, Adv. Mater. 2012, 24, 2047.

[48] N. Li, S. X. Jin, Q. Y. Liao, C. X. Wang, ACS Appl. Mater. Inter. 2014, 6, 20590.

[49] Y. Sharma, N. Sharma, G. V. Subba Rao, B. V. R. Chowdari, Adv. Funct. Mater. 2007, $17,2855$.

[50] X. Sun, C. Zhou, M. Xie, H. Sun, T. Hu, F. Lu, S. M. Scott, S. M. George, J. Lian, J. Mater. Chem. A 2014, 2, 7319.

[51] Z. Zhu, S. Wang, J. Du, Q. Jin, T. Zhang, F. Cheng, J. Chen, Nano Lett. 2014, 14, 153.

[52] G. Xu, J. Li, L. Huang, W. Lin, S. G. Sun, Nano Energy 2013, 2, 394.

[53] Y. Dou, J. Xu, B. Ruan, Q. Liu, Y. Pan, Z. Sun, S. X. Dou, Adv. Energy Mater. 2016, $6,1501835$. 


\section{WILEY-VCH}

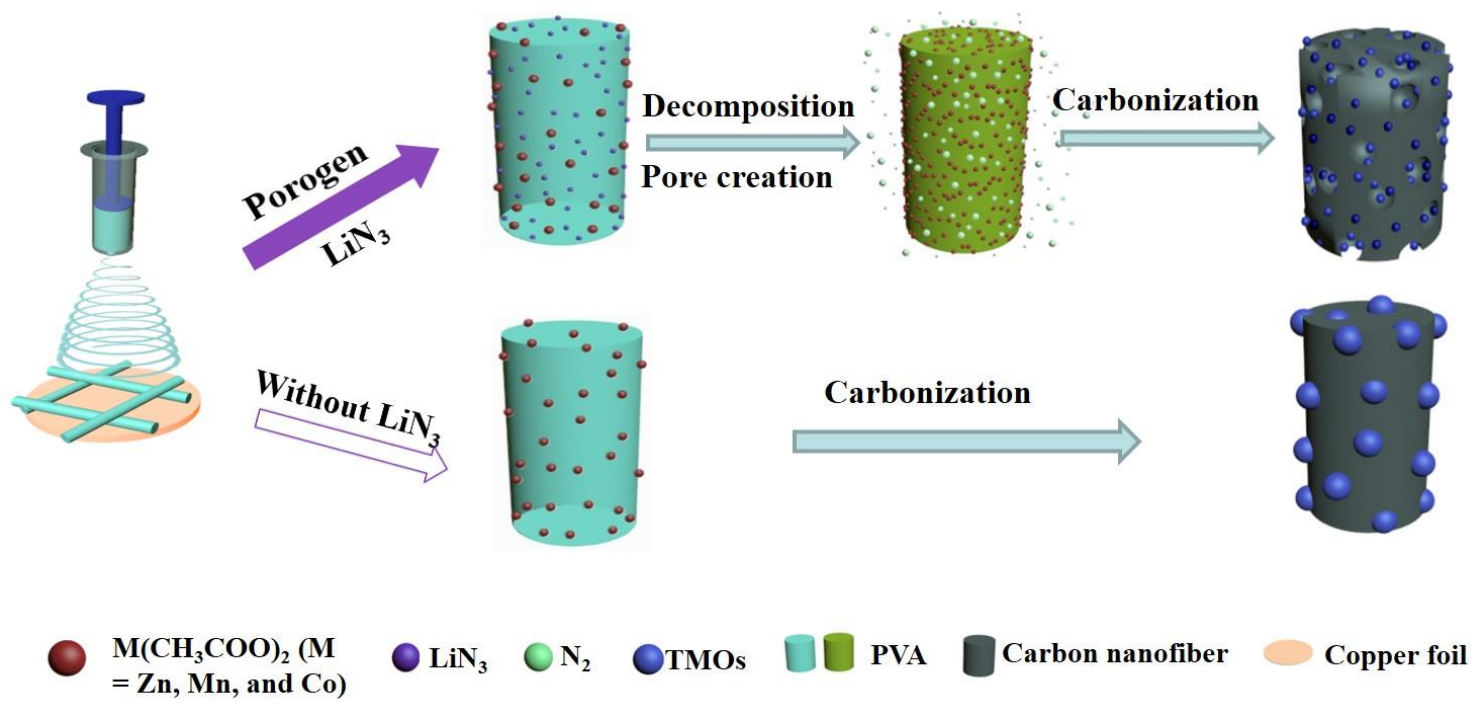

Figure 1. Schematic illustration of the formation process for TMOs UNPs@HPCNFs $(M=$ $\mathrm{Zn}, \mathrm{Mn}$, and Co). 


\section{WILEY-VCH}

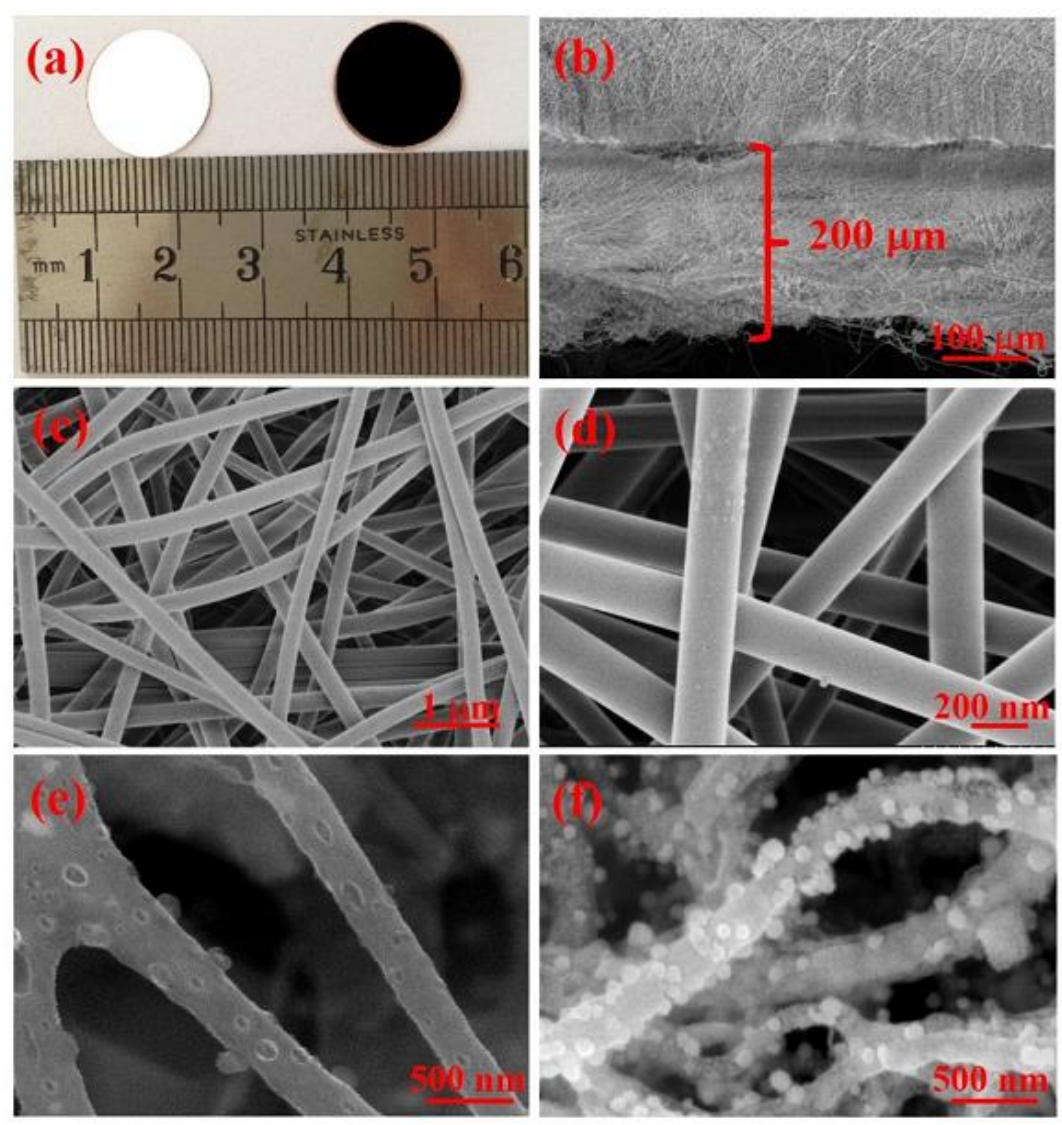

Figure 2. (a) Digital images of the as-electrospun nanofibers and the as-prepared TMOs UNPs@HPCNFs. (b) Cross-sectional SEM image of TMOs UNPs@HPCNFs. Typical SEM images of the obtained TMOs UNPs@HPCNFs before (c and d) and after (e) carbonization at different magnifications. (f) SEM image of ZnO NPs@CNFs. 


\section{WILEY-VCH}
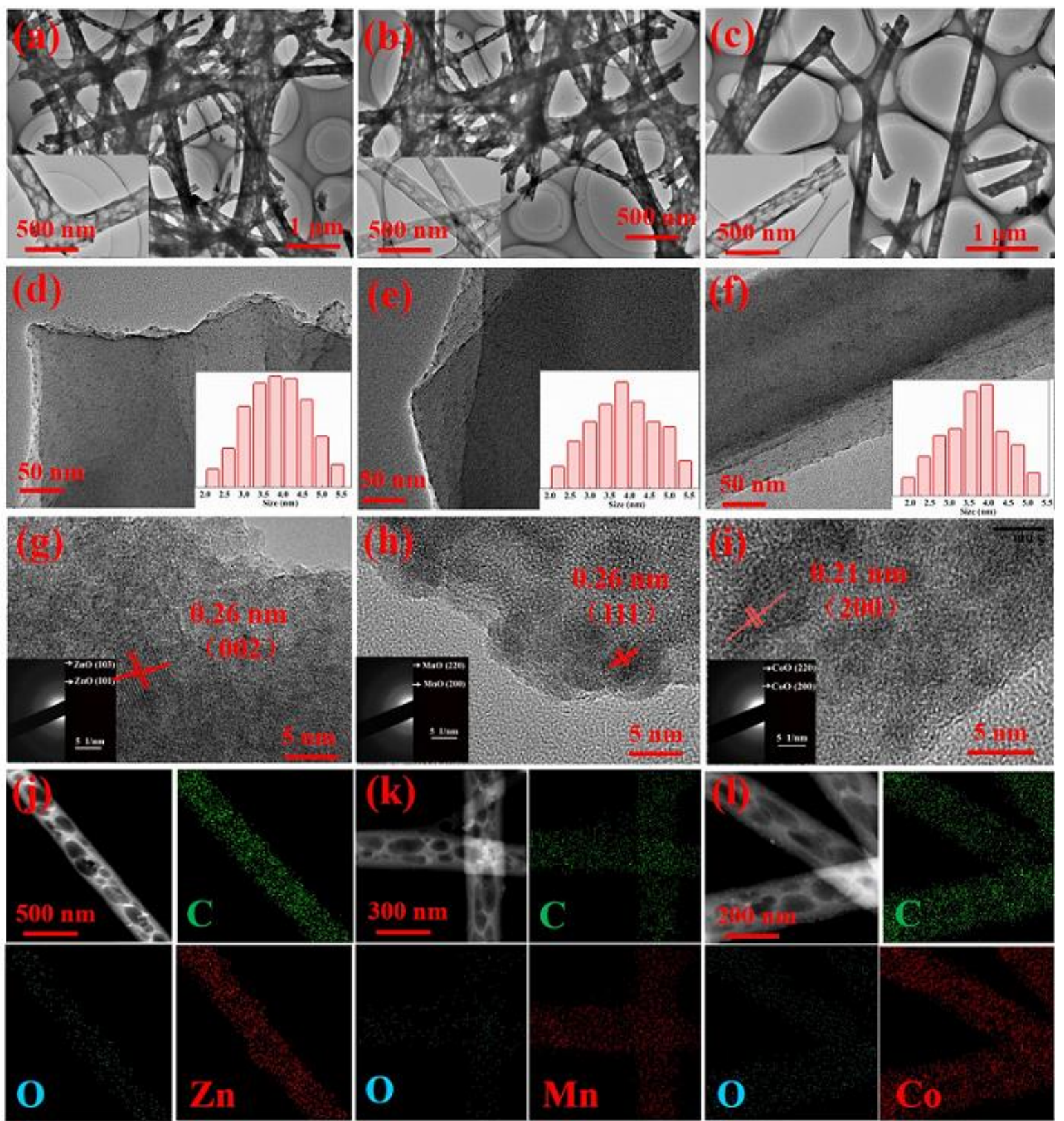

Figure 3. TEM images of the as-prepared (a and g) ZnO UNPs@ HPCNFs, (b and e) MnO UNPs@HPCNFs, and (c and f) CoO UNPs@HPCNFs. Magnified TEM images of (d) ZnO UNPs@HPCNFs, (e) MnO UNPs@HPCNFs, and (f) CoO UNPs@HPCNFs, with the insets showing the quantum dot size distributions. HRTEM images of ( $\mathrm{g}$ ) ZnO UNPs@ HPCNFs, (h) MnO UNPs@HPCNFs, and (i) CoO UNPs@HPCNFs. (Insets of a, b, and c show higher magnification, while insets of $\mathrm{g}$, $\mathrm{h}$, and $\mathrm{i}$ are the corresponding SAED patterns.) STEM and the corresponding elemental mapping of (j) ZnO UNPs@HPCNFs, (k) MnO UNPs@HPCNFs, and (1) CoO UNPs@HPCNFs. 


\section{WILEY-VCH}

(a)
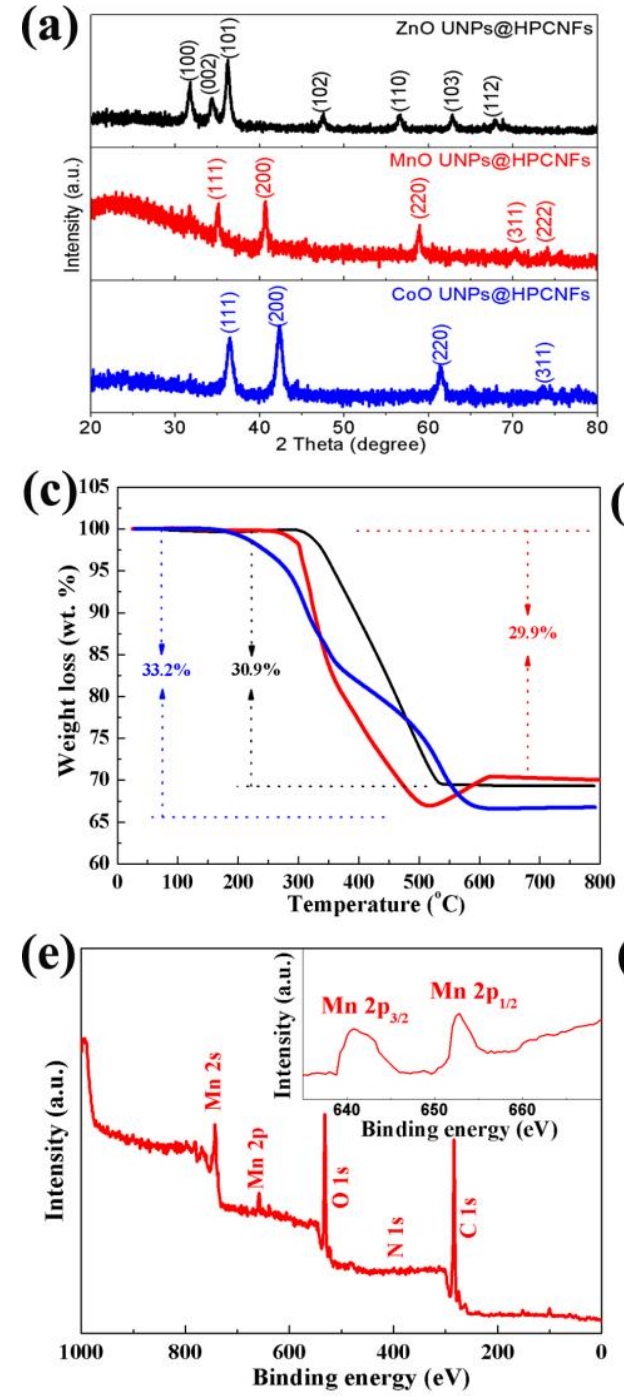
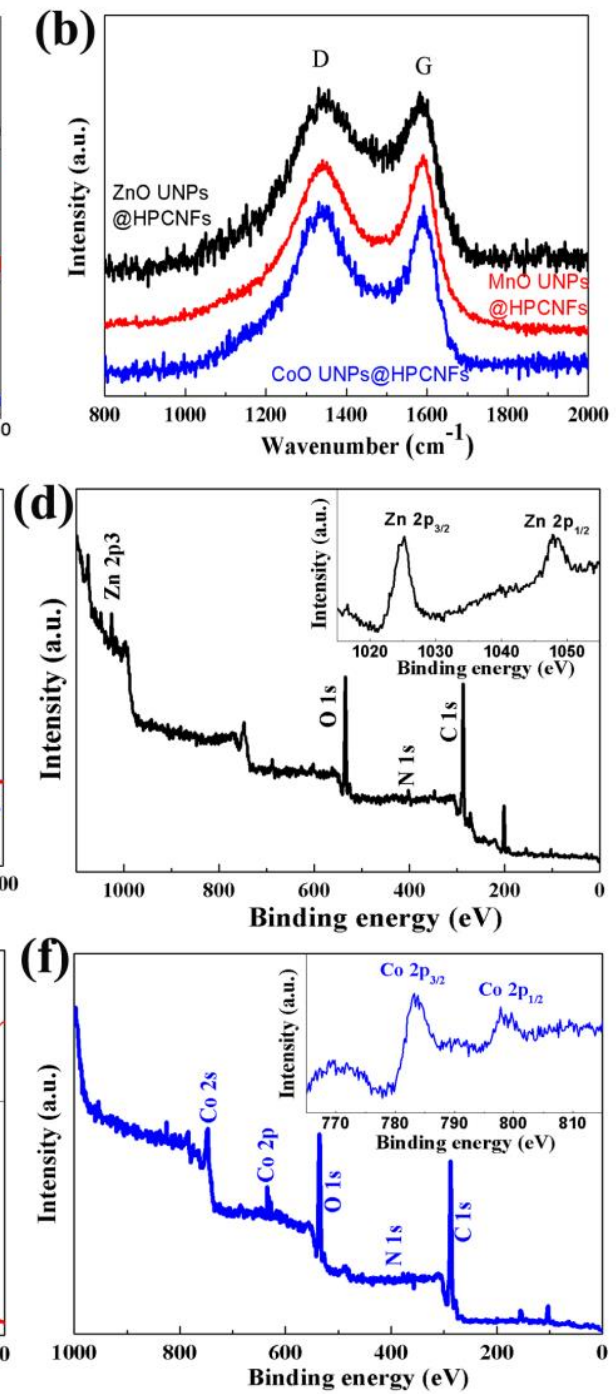

Figure 4. (a) XRD patterns, (b) Raman spectra, and (c) TGA results for the as-prepared TMOs UNPs@HPCNFs.XPS results of (d) ZnO UNPs@HPCNFs, (e) MnO

UNPs@HPCNFs, and (f) CoO UNPs@HPCNFs, with the insets showing enlarged spectra of the indicated regions. 


\section{WILEY-VCH}
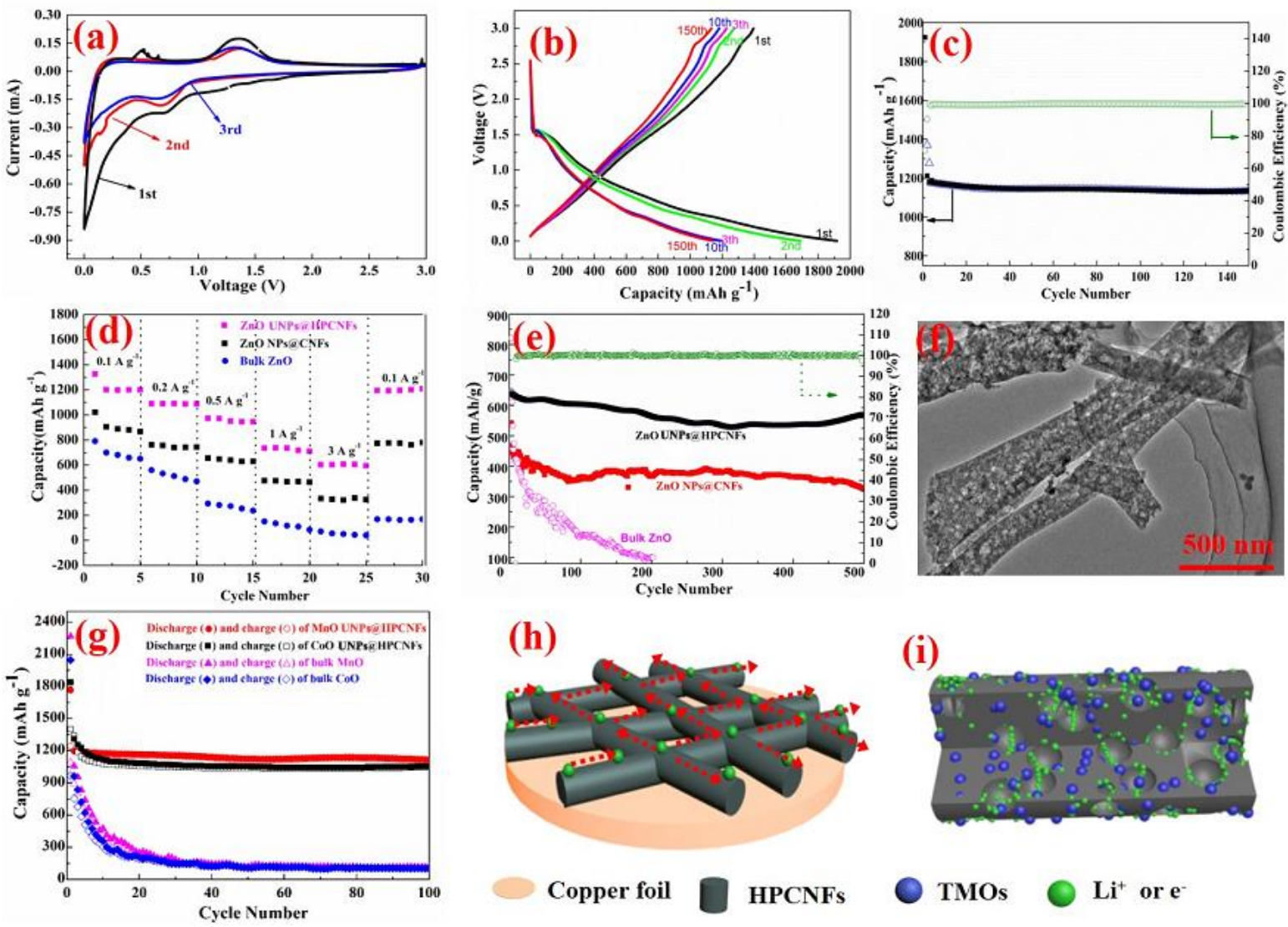

(h)

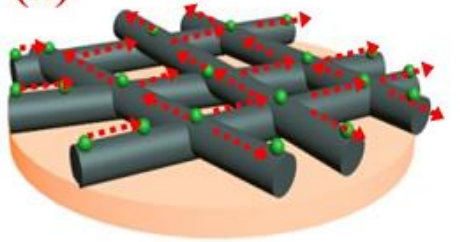

Copper foil (i)

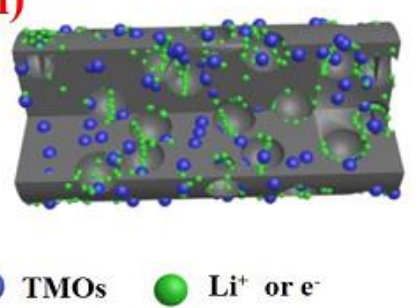

Figure 5. (a) CV curves for the initial three cycles of ZnO UNPs@HPCNFs at a scan rate of $0.1 \mathrm{mV} \mathrm{s}^{-1}$. (b) Galvanostatic charge/discharge curves of ZnO UNPs@HPCNFs at a cycling rate of $0.1 \mathrm{~A} \mathrm{~g}^{-1}$ for the $1^{\text {st }}, 2^{\text {nd }}, 3^{\text {rd }}, 10^{\text {th }}$ and $150^{\text {th }}$ cycles. (c) Charge/discharge capacity and coulombic efficiency of ZnO UNPs@HPCNFs electrode versus cycle number at a current density of $0.1 \mathrm{~A} \mathrm{~g}^{-1}$. (d) Cycling and rate capacities of ZnO UNPs@ HPCNFs, ZnO NPs@CNFs, and bulk ZnO at various current densities from 0.1 to $3 \mathrm{~A} \mathrm{~g}^{-1}$. (e) Charge/discharge capacity and coulombic efficiency of ZnO UNPs@HPCNFs electrode versus cycle number at a cycling rate of $3 \mathrm{~A} \mathrm{~g}^{-1}$, including ZnO NPs@CNFs and bulk ZnO for comparison. (f) TEM image of ZnO UNPs@HPCNFs after 150 cycles at a cycling rate of $3 \mathrm{~A}$ $\mathrm{g}^{-1}$. (g) Charge/discharge capacity of MnO UNPs@HPCNFs and CoO UNPs@ HPCNFs electrodes versus cycle number at a cycling rate of $0.1 \mathrm{~A} \mathrm{~g}^{-1}$, including bulk $\mathrm{MnO}$ and $\mathrm{CoO}$ for comparison. (h and i) Schematic illustration of the structural advantages of TMOs UNPs@HPCNFs as anode electrode materials. 


\section{WILEY-VCH}

A unique general, large-scale, and cost-effective strategy is demonstrated towards fabricating various transition metal oxides into ultrafine nanoparticles (TMOs UNPs) uniformly embedded in hierarchically porous carbon nanofibers. Such unique nanostructures efficiently avoid the aggregation and pulverization of TMOs, promote the rapid threedimensional transport of both $\mathrm{Li}$ ions and electrons, and enhance the electrical conductivity and structural integrity, rendering a significant improvement in electrochemical activities for lithium storage.

Keyword: electrospinning, porous carbon nanofibers, transition metal oxide, nanoparticles, lithium-ion batteries.

G. L. Xia, L. J. Zhang, F. Fang, D. L. Sun, Z. P. Guo, H. K. Liu, X. B. Yu*

General Synthesis of Transition Metal Oxide Ultrafine Nanoparticles Embedded in Hierarchically Porous Carbon Nanofibers as Advanced Electrodes for Lithium Storage

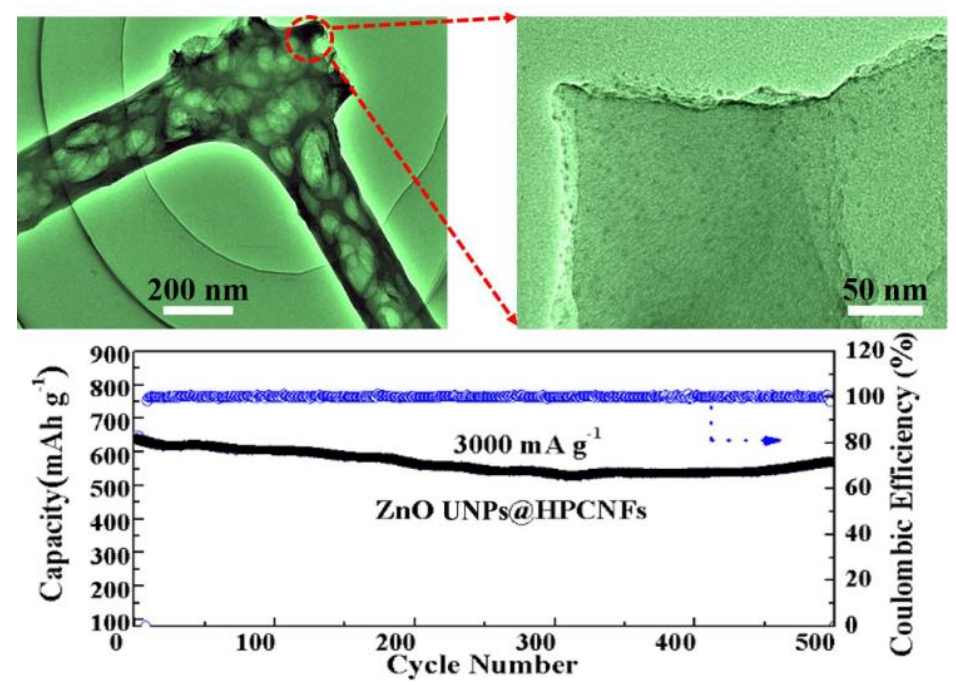

